Pharmacists were mostly female (63\%), and Caucasian (66\%). Most were HIV-certified (68\%); 31\% worked in specialty-only and $21 \%$ in traditional-only pharmacies. Majority were comfortable discussing HIV (91\%), condom use (91\%), and counselling PLWH who were heterosexual (96\%) or MSM (97\%). However, 33\% were uncomfortable selling PWIDs needles and $48 \%$ teaching PWIDs to use clean needles. HIV-certified pharmacists were twice as likely to be comfortable selling PWIDs needles $\left(\mathrm{OR}_{\text {sellneedles }} 2.46 ; \mathrm{p}<0.001\right)$ than condom use counselling $\left(\mathrm{OR}_{\text {counselcondomuse }} 1.10 ; \mathrm{p}<0.001\right)$. Pharmacist comfort-level discussing HIV increased by $2 \%$ with age.

Conclusion Our finding that a significant proportion of pharmacists were uncomfortable serving PWIDs is concerning given that pharmacists might have frequent encounters with this hard-toreach vulnerable population. Continuing professional education (CPE) curricula should be expanded to improve pharmacists' ability and comfort serving populations at high-risk for HIV.

Disclosure statement This study was funded by the National Institutes of Mental Health, U. S. A. No pharmaceutical grants were received in the development of this study.

\section{P18.09 COMMUNITY-BASED SUPPORT GROUPS ENGAGEMENT IN HIV PREVENTION AND ECONOMIC EMPOWERMENT IN RURAL KENYA}

Kibicho Jennifer ${ }^{*}$, Penninah M Kako, Patricia E Stevens. College of Nursing, University of Wisconsin-Milwaukee, Milwaukee, WI, USA

\subsection{6/sextrans-2015-052270.632}

Background Support groups' important role in destigmatizing HIV/AIDS and addressing social-psychological needs of persons living with HIV (PLWH) is well documented. However, the impact of support groups in economic activities is less studied. Our study compares HIV- and non-HIV- support groups in providing social-psychological and economic empowerment for PLWH or at-risk groups in rural Kenya.

Methods We recruited 72 participants in 12 support groups (42 women, 30 men; 6 HIV, 6 non-HIV). Interviews lasted 1.5-4 h. Participants were asked to describe the benefits and challenges of support groups. We used MAXQDA qualitative software. We used thematic analysis informed by Grounded Theory principles to develop themes.

Results HIV- and non-HIV groups provided socio-psychological benefits to members. However, HIV groups engaged in community-based HIV testing promotion, and empowered members on fight HIV-stigma and to disclose HIV-status. HIV-group$s$ - mostly donor-financed-had challenges related to financial mismanagement and lack of transparency of disbursements.

Non-HIV groups - mostly micro-financing initiatives - offered financial assistance (e.g., school fees, hospital expenses), start-up capital for business projects, and improved the standard of living for members. Non-HIV-groups had challenges related to marital conflict and violence about finances, high rate of loan-default among members; and high direct (e.g., membership financial contribution, transport costs) and indirect (e.g., time commitments) costs of group participation.

Conclusion HIV-support groups are underutilised in economic empowerment initiatives for PLWH, and face challenges of sustainability, in part due to poor leadership, financial mismanagement and high financial dependency on external funding. NonHIV groups are underutilised as venues for community-based HIV prevention efforts. Before engaging in micro-finance activities, support groups should receive leadership and financial training to ensure their long-term sustainability and increase group effectiveness in uplifting the quality and standards of living for persons living with or at-risk for HIV in rural communities.

Disclosure of interest statement This study was funded by the University of Wisconsin-Milwaukee College of Nursing Start-Up funds, U. S. A. No pharmaceutical grants were received in the development of this study.

\section{P18.10 DETERMINANTS OF QUALITY OF LIFE AMONG PEOPLE LIVING WITH HUMAN IMMUNODEFICIENCY VIRUS (PLHIV) IN COASTAL SOUTH INDIA}

Bhaskaran Unnikrishnan*. Kasturba Medical College (Manipal University) Mangalore, Karnataka, India

\subsection{6/sextrans-2015-052270.633}

Aim To assess the quality of life (QOL) of PLHIV and to identify the factors influencing their QOL.

Method A cross-sectional study was conducted among 302 PLHIV aged 18 years and above, attending ART centre of Kasturba Medical College Hospital, Mangaluru, India from April to December 2014. Institutional Ethics Committee (IEC) approval was obtained prior to the commencement of the study. After obtaining a written informed consent, PLHIV were interviewed using WHOQOL-HIV BREF questionnaire to assess their QOL. Data was entered in, and analysed using SPSS version 16.

Results The mean scores (SD) across the six domains of QOL were physical- 16.37 (2.18); psychological- 12.40 (2.02); level of independence- 13.56 (2.28); social relationship- 12.19 (1.69); environment- 12.37 (2.03) and spirituality- 12.42 (2.23). A statistically significant difference was observed between the various domain scores of QOL (psychological; level of independence; social relationship; environment and spirituality) with socio economic status (SES) of PLHIV $(\mathrm{P}<0.05)$ except the physical domain $(\mathrm{P}>0.05)$. A significant difference was also observed between psychological domain of QOL among PLHIV and the presence of opportunistic infection. $(\mathrm{P}=0.028)$

Conclusion In our study, poor SES and presence of opportunistic infection have an adverse effect on QOL among PLHIV receiving ART.

\section{P18.11 EXAMINING THE EFFECT OF CASE MANAGEMENT ON LEVELS OF DEPRESSION AMONG NEWLY DIAGNOSED PEOPLE LIVING WITH HIV IN TAIWAN}

CT Yu*, MC Feng, LH Chen, WH Wen, SF Liu, YC Chuang. Kaohsiung Medical University Hospital, Kaohsiung Medical University, Nursing, Kaohsiung, Taiwan, Province of China

\subsection{6/sextrans-2015-052270.634}

Introduction Over half of Taiwan's HIV patients have been enrolled in case management. One of goals of HIV case management is to provide physical and mental consultations for the clients' need. The prevalence of depression in people living with HIV (PLWHA) is higher than that in the general public and possibly leads to worse HIV related outcomes. The aims of this study were to evaluate the efficacy of PLWHA in case management services so as to improve any depression and/or related symptoms. 